\title{
Isolation and characterization of microcrystalline cellulose from oil palm biomass residue.
}

\begin{abstract}
In this work, we successfully isolated microcrystalline cellulose (MCC) from oil palm empty fruit bunch (OPEFB) fiber-total chlorine free (TCF) pulp using acid hydrolysis method. TCF pulp bleaching carried out using an oxygen-ozone-hydrogen peroxide bleaching sequence. Fourier transform infrared (FT-IR) spectroscopy indicates that acid hydrolysis does not affect the chemical structure of the cellulosic fragments. The morphology of the hydrolyzed MCC was investigated using scanning electron microscopy (SEM), showing a compact structure and a rough surface. Furthermore, atomic force microscopy (AFM) image of the surface indicates the presence of spherical features. X-ray diffraction (XRD) shows that the MCC produced is a cellulose-I polymorph, with $87 \%$ crystallinity. The MCC obtained from OPEFB-pulp is shown to have a good thermal stability. The potential for a range of applications such as green nano biocomposites reinforced with this form of MCC and pharmaceutical tableting material is discussed.
\end{abstract}

Keyword: Oil palm empty fruit bunch; Pulp bleaching; Cellulose; Microcrystalline cellulose. 